X-ray scattering intensities of water at extreme pressure and temperature

N. Goldman, L. E. Fried

January 3, 2007

Journal of Chemical Physics 
This document was prepared as an account of work sponsored by an agency of the United States Government. Neither the United States Government nor the University of California nor any of their employees, makes any warranty, express or implied, or assumes any legal liability or responsibility for the accuracy, completeness, or usefulness of any information, apparatus, product, or process disclosed, or represents that its use would not infringe privately owned rights. Reference herein to any specific commercial product, process, or service by trade name, trademark, manufacturer, or otherwise, does not necessarily constitute or imply its endorsement, recommendation, or favoring by the United States Government or the University of California. The views and opinions of authors expressed herein do not necessarily state or reflect those of the United States Government or the University of California, and shall not be used for advertising or product endorsement purposes. 


\title{
X-ray scattering intensities of water at extreme pressure and temperature
}

\author{
Nir Goldman* and Laurence E. Fried
}

Lawrence Livermore National Laboratory, Chemistry Materials and Life Sciences Directorate, L-268, Livermore, California 94551

\begin{abstract}
We have calculated the coherent x-ray scattering intensity of several phases of water at 1500 and 2000 K under high pressure, using ab initio Density Functional Theory (DFT). Our calculations span the molecular liquid, ice VII, and superionic solid phases, including the recently predicted symmetrically hydrogen bonded region of the superionic phase. We show that wide angle $\mathrm{x}$-ray scattering intensity could be used to determine phase boundaries between these high pressure phases, and we compare the results for ice VII and superionic water. We compute simulated spectra and provide new atomic scattering form factors for water at extreme conditions, which take into account frequently neglected changes in ionic charge and electron delocalization. We show that our modifed atomic form factors allow for a nearly exact comaprison to the total x-ray scattering intensities calculated from DFT. Finally, we analyze the effect our new form factors have on determination of the oxygen-oxygen radial distribution function.
\end{abstract}

\section{Introduction}

Detailed knowledge of the chemistry of water at extreme conditions (hundreds of GPa and thousands of Kelvin) is imperative to understanding earth and planetary sciences, and the chemical reactivity that occurs within such hot, compressed systems. Currently there is little scientific knowledge about reaction pathways and the nature of the molecular to non-molecular transition at high temperature and pressure. It is not even clear if conventional chemical notions, such as covalent vs. ionic bonding, still hold at these conditions.

Despite recent technological advances, molecular and atomic scale information is still difficult to obtain experimentally, and theoretical studies are necessary. Phase diagram information is vital for equation of state

*corresponding author: goldman14@1lnl.gov 
determination; joint expeirmental and computational studies could lead to the discovery of new material properties. In one such example, recent results by Goncharov et al. [1] and Goldman et al. [2] have confirmed the presence of superionic water, an exotic phase wherein the oxygens have formed a stable body-centered-cubic (bcc) lattice and the hydrogens dissociate freely and diffuse at liquid-like rates. Superionic water occurs at conditions similar to the interiors of Neptune and Uranus, and could explain the high magnetic field measurements of these planets by the Voyager 2 spacecraft. In the superionic phase $\mathrm{O}-\mathrm{H}$ bond dissociation occurs at such rapid rates that conventional definitions of a chemical bond are inadequate [2,3], and water forms a distinctly "non-molecular" phase which is best described as an ensemble of transition states. Nonetheless, the high pressure phase diagram of water is not well defined, including the boundaries of the superionic phase, ice VII, VIII, and the symmetrically hydrogen-bonded ice X. In addition, our recent results suggest that exotic phases, like the superionic solids of hydrogen bonding compounds, occur during the detonation of high explosives [1-3].

In principle, accurate structural determination of the phases of water can be determined from scattering expeirments [4]. In water, the x-ray scattering is dominated by oxygen-oxygen correlations, because most of the electron density of the molecules is centered around the oxygen atoms. X-ray scattering has the advantage over neutron scattering in that the data does not need to be massively corrected before conversion to real space [4-7]. In addition, due to recent advances in $a b$ initio codes, $x$-ray scattering spectra can be readily calculated using a combination of all electron methods and density functional theory $[6,7]$. The $x-$ ray scattering intensity, $I(Q)$, is entirely dependent upon the electron density of the molecules of interest. As mentioned above, as pressures and temperatures are increased to the conditions of planetary interiors, the molecular electron density is expected to change drastically, as bonding becomes symmetric, as in the case of ice X, or exceedingly short, as in the case of superionic water. The $\mathrm{x}$-ray scattering intensity serves as a sensitive probe the effects of pressure and temperature on condensed matter, such as water. X-ray scattering spectra consequently can allow for clear determination of phase boundaries, and provide very clear ways to compare theory and experiment. A number of x-ray studies of ice VII [8-10], ice VIII [11] and ice X [12] exist. It has also been suggested that ice VII might exist in cold conducting slabs within the Earth's mantle [13]. However, to date no x-ray study has probed the elevated temperatures at or around the superionic phase of water. Thus the ability of x-ray diffraction to study high pressure-temperature phases of water, including the superionic phase, has yet to be investigated.

Through the use of atomic scattering form factors, $f(Q)$, discussed below, the structure of the particular phase of water can be determined, such as the oxygen-oxygen radial distribution function, $g\left(R_{O O}\right)$, or RDF. The $g\left(R_{O O}\right)$, provides a simple and direct way to compare simulation and experiment. Such a comparison a comparison is much simpler and easier to perform than direct calculation of the x-ray scattering intensity, 
which can require use of expensive ab initio codes and supercomputers (see below). In addition, accurate determination of the RDF has the ability to greatly facilitate empirical force field development. Thus it is the goal of this work to not only provide accurate determination of x-ray scattering intensities, but also to aid in the experimental determination of the $g\left(R_{O O}\right)$ at extreme pressures and temperatures.

We present herein calculations of the total x-ray scattering intensity of water at 1500 and $2000 \mathrm{~K}$, under high pressure conditions, using ab initio density functional theory. We show that the $I(Q)$ clearly indicates the molecular liquid to superionic solid phase transition in water at high temperature and pressure. In addition, we compare the $I(Q)$ results for superionic water and ice VII. We also determine new atomic form factors which more accurately reproduce the total x-ray scattering intensity, compared to the standard atom-centered electron density approximation. We show that our new form factors can provide peak intensities of the $I(Q)$ that are $10-15 \%$ higher. However, the effect appears to rather minimal on the determination of the $g\left(R_{O O}\right)$. Finally we analyze the effect of high pressure and temperature on electron delocalization and ionic charge.

\section{Method}

The formalism for determination of $I(Q)$ and $g\left(R_{O O}\right)$ has been reviewed elsewhere $[4,6,7,14]$ and is briefly included herein for the sake of completeness. The x-ray scattering of an electron density generated form a condensed phase simulation trajectory can be calculated via the following equation:

$$
I(Q)=\left\langle|F(Q)|^{2}\right\rangle
$$

where

$$
F(Q)=\int_{0}^{\infty} \rho(r) \exp (i Q \cdot r) d r
$$

is the Fourier Transform of the one electron density, $\rho(r)$. Frequently, the total electron density is approximated as a superposition of electron densities centered on individual atoms. Consequently, the total x-ray scattering intensity can be calculated via direct sum over atomic positions [14]:

$$
I(Q)=\left\langle\sum_{i} \sum_{j} f_{i}(Q) f_{j}(Q) \exp \left(i Q \cdot r_{i j}\right)\right\rangle .
$$

Here, the $f(Q)$ correspond to the atomic scattering factors [15], and the $r_{i j}$ are the vectors connecting interatomic sites (both intra- and intermolecular). The exponential in Eqn. 3 corresponds to the atomic structure 
factor, viz.,

$$
S(Q)=\frac{1}{N} \sum_{i} \sum_{j} \exp \left(i Q \cdot r_{i j}\right)
$$

Standard values of $f(Q)$ used in determination of the $I(Q)$ are calculated for the isolated atoms. However, this approximation is flawed in that as atoms come together to form covalent bonds, the charge density on each atom is clearly changed [4]. The effect is likely to be even more pronounced at high pressure-temperature conditions, where molecular bonding changes dramatically, causing the formation of new classes of materials [16]. With recent advances in ab initio codes [6,17], it has become possible but costly to directly calculate the total $\mathrm{x}$-ray scattering intensity of a condensed phase simulation via Fourier Transform of the one-electron density. We are thus in a unique position to assess the efficacy of standard atomic form factors and to determine more accurate values for high pressures and temperatures. This is particularly relevant considering the rapid pace at which diamond anvil cell techniques are able to achieve extreme pressures and temperatures [1], and could soon perform x-ray scattering experiments near superionic water conditions. Through prediction of accurate form factors, we can greatly facilitate comparison between theory and experiment, namely via the radial distribution function. The $g(R)$ is related to the $S(Q)$ via Fourier Transform [18].

In our calculations we have used molecular dynamics trajectories generated for previous publications [1, 2]. In summary, trajectories were generated by the CPMD code v.3.9.1, with the BLYP exchange-correlation functional [19,20], and Troullier-Martins pseudo-potentials [21] for both oxygen and hydrogen. A planewave cutoff of 120 Ry was employed to insure convergence of the pressure, although all other properties were seen to converge with a much lower cutoff ( $85 \mathrm{Ry}$ ). The system size was $54 \mathrm{H}_{2} \mathrm{O}$ molecules, and all trajectories were run for 5-10 ps. The temperature was controlled by using Nosé-Hoover thermostats [22] for all nuclear degrees of freedom. The importance of an appropriate choice of electron mass in Car-Parrinello molecular dynamics of water has been pointed out in recent studies [23,24]. We chose a conservative value of $200 \mathrm{au}$, and a time step of $0.048 \mathrm{fs}$. Initial conditions for the $1500 \mathrm{~K}$ trajectory were generated by compressing a liquid configuration at $1.0 \mathrm{~g} / \mathrm{cc}$ to the desired density in sequential steps of up to $0.2 \mathrm{~g} / \mathrm{cc}$. Initial conditions for the $2000 \mathrm{~K}$ trajectory were generated by relaxing an ice VII configuration at the density of interest, and heating to $2000 \mathrm{~K}$ via velocity scaling in sequential steps of 300 degrees for $0.5-1 \mathrm{ps}$. At high pressure, in general the $1500 \mathrm{~K}$ trajectory exhibited a superionic solid wherein the oxygens were quenched into a metastable disordered state, due to hysteresis, where an underlying bcc lattice is the free energy minimum. In one case, discussed below, the oxygens spontaneously formed a bcc lattice. Conversely, the $2000 \mathrm{~K}$ trajectory exhibited a superionic phase wherein the oxygens maintained a stable bcc lattice throughout the simulation.

Krog-Moe [25] and Norman [26] have described a method for normalizing experimental x-ray scattering intensities to an absolute scale. The method defines the scale factor $\alpha$ as follows: 


$$
\alpha=\frac{-2 \pi^{2} z^{2} \rho+\int_{0}^{Q_{0}} Q^{2} d Q\left[f^{2}(Q)+I_{\text {incoherent }}(Q)\right]}{\int_{0}^{Q_{0}} Q^{2} d Q I_{\text {experiment }}(Q)},
$$

where $z$ is the number of electrons per water molecule, $\rho$ is the number density of the system (in molecules $/ \AA^{3}$ ), $Q_{0}$ is the maximum value of momentum transfer available in the experiment, $f(Q)$ is the molecular form factor for water, $I_{\text {incoherenet }}(Q)$ is the incoherent or Compton scattering from the sample (discussed below), and $I_{\text {experiment }}(Q)$ is the experimental spectrum to be normalized. The Compton scattering for a single water molecule has been calculated previously [27, 28]. However, given the exceedingly short lifetimes of water at the conditions of our calculations, such a calculation is unlikely to be accurate. Thus, we have chosen to neglect this term from our normalization. In addition, Hura et al. state that the energy of a Compton event is energy of the beam used in their experiment [5]. Thus, it is unlikely that inclusion of the Compton scattering will have significant impact on the normalization. Molecular form factors for water at lower temperature and pressure are available (e. g., Ref. [29]), but similarly are not applicable to the extreme thermodynamic conditions of this work. As a result, we have chosen to normalize to the form factor calculated by the independent atomic scattering method (IAM) [29]. Ultimately, the choice of parameters for normalization are unimportant for the scope of this work., since we have focused our efforts on comparing $I(Q)$ calculated by different methods, and on the determination of improved atomic scattering factors.

The total x-ray scattering intensity from the ab initio electron density, $I_{e l}(Q)$, was calculated via the QUICKSTEP code, which is part of the CP2K molecular dynamics suite [17,30]. As stated in [6], plane wave pseudopotentials are not well suited to describing the electron density near the nuclei. Thus the allelectron version of the code was used. We used a basis set of TZVP for the oxygens and DZVP for the hydrogens. All of our calculations used the BLYP exchange correlation functional, as well. The smooth part of the electron charge density was was expanded to an energy cutoff of $130 \mathrm{Ry}$, as conducted previously [6]. Calculations were performed with cutoffs up to $200 \mathrm{Ry}$ with almost no visible difference in the calculated $\mathrm{x}$-ray scattering spectrum. Snapshots from our CPMD simulations were fed into the CP2K code in intervals of ca. $2.5-5 \mathrm{fs}$.

\section{Results}

We first present results for $I_{e l}(Q)$ calculated for trajectories along the $1500 \mathrm{~K}$ isotherm (Fig. 1). In contrast to the experimental and theoretical results for the total x-ray scattering intensity at ambient conditions [5-7], the double peak normally seen at ca. $2-3 \AA^{-1}$ has been replaced by a single, much narrower peak. The results for 12 and 44 GPa clearly show a broad, liquid like structure, which is consistent with previous finding in which 
a liquid phase was observed at these conditions [1]. At $44 \mathrm{GPa}, I_{e l}(Q)$ appears to be forming a doublet, likely due to the fact that the simulation is near the liquid-superionic solid transition. At $48 \mathrm{GPa}$, we observe a bcc lattice, as evidenced by the spacing and narrowness of the scattering peaks. As previously reported [1], we observe the onset of the superionic phase at these conditions. The spontaneous crystallization is quite remarkable, given the initial disordered configuration of the simulation. The fact that an oxygen bcc lattice is spontaneously formed lends credence to the idea that the superionic phase is the free energy minimum at these conditions. However, in general, simulations of the superionic phase beginning from a disordered configuration formed an amorphous oxygen phase with a rapidly diffusing hydrogen phase.

Results for the $2000 \mathrm{~K}$ isotherm are shown in Figure 2. At $34 \mathrm{GPa}$, we again observe the reactive molecular liquid, in which previous simulation had shown a predominance of $\mathrm{H}_{2} \mathrm{O}$ molecules with short lifetimes (ca. $40 \mathrm{fs}$ ) [2,3]. At $46 \mathrm{GPa}$, we still observe the reactive molecular liquid, albeit once again the presence of a doublet could be due to proximity of the superionic phase boundary. At $58 \mathrm{GPa}$ we observe a stable bcc lattice of oxygen ions, hence, the onset of the superionic phase. This is in contrast to our original findings $[1,2]$ based on the oxygen diffusion constant, in which we saw the onset of the asymmetric hydrogen bonded superionic phase at $75 \mathrm{GPa}$. This phase is one in which the covalent and ionic bonds are still distinct, viz., the covalent bonds are significantly shorter than the ionic. The oxygen diffusion constant appeared to be small but greater than zero at $58 \mathrm{GPa}$, which was likely the result of the short length of the trajectory. Clearly, the total $\mathrm{x}$-ray scattering intensity serves as a much more precise order parameter for this phase transition. At $115 \mathrm{GPa}$, we observe the symmetrically hydrogen bonded superionic phase. In this case, the covalent and ionic bonds have become approximately equivalent. As seen in Fig. 2, the scattering intensity for this state point is nearly identical to those from the other superionic, asymmetrically hydrogen bonded simulations (58 and $75 \mathrm{GPa}$ ). Hence, the x-ray scattering intensity alone cannot not determine the exact chemical nature of water at those conditions, and molecular interpretation of experiments will have to rely of simulations for simple chemical pictures.

In an effort to observe a distinct experimental result that could distinguish between the superionic phase and a low temperature ice (viz., ice VII or ice X) in which the hydrogens are non-diffusive, we have computed the $I_{e l}(Q)$ for both symmetric hydrogen bonded superionic water (115 GPa and $2000 \mathrm{~K}$ ) and ice VII (94 GPa and $300 \mathrm{~K})$. The results are shown in Fig. 3. The main difference between the two spectra is that ice VII exhibits larger peak intensities than the superionic phase. In addition, the structure factors and standard atomic form factors for oxygen and hydrogen can be used to analyze the intensity from the 111 reflection $\left(I_{111}\right)$. This peak is of particular interest since it has been reported that over $99 \%$ of its intensity in ice VII and $\mathrm{X}$ is due to the hydrogens alone [12]. Thus this peak provides a unique picture of the state of the hydrogen atoms at these conditions. We have determined that the hydrogen-hydrogen scattering comprises ca. $85 \%$ 
of $I_{111}$ for our ice VII simulation, compared to $25 \%$ for the superionic solid. This is a sharp decrease as a function of temperature, and is likely due to the dramatic increase in hydrogen mobility upon formation of the superionic phase. This type of analysis could provide a method for direct experimental observation of the superionic solid. However, $I_{111}$ occurs at relatively low values of $Q$, where standard atomic form factors are most prone to error, particularly at high pressure and temperature.

Use of standard atomic form factors assume a spherical electron charge density is placed on each atom $[4,7]$. These charge values are based on the isolated atoms and consequently ignore the effects of chemical bonding, viz., contraction and/or dilation of the valence band. The standard atomic form factors were found to be inaccurate for calculations of ambient water, wherein the first peak and shoulder were were calculated at too low intensity. In order to modify the form factors, Sorenson et al. [4] introduced the following:

$$
f^{\prime}(Q)=\left[1+(\alpha-1) \exp \left(-Q^{2} / 2 \delta^{2}\right)\right] f(Q)
$$

where $f^{\prime}(Q)$ is the modified atomic form factor (MFF), $f(Q)$ is the standard atomic form factor (same as above), $\alpha$ is a scaling factor representing the redistribution of charge, in this case specifically on the oxygens since they comprise $85 \%$ or greater of the intensity of the $I(Q)$. The $\delta$ is a fitted parameter representing the valence electron delocalization caused by the formation of chemical bonds. By choosing a value of $\alpha$ equivalent to a liquid dipole moment of $2.8 \mathrm{D}$ and a value of $\delta=2.2 \AA^{-1}$, Sorenson et al. were able to observe improved agreement between the experimental and theoretical x-ray scattering intensities [4]. Similarly, we have used a Levenberg-Marquardt non-linear least squares fitting routine [31] to fit the parameters of Eqn. 6 to our $I_{e l}(Q)$ data at 1500 and $2000 \mathrm{~K}$. Our results, labeled $I_{M F F}(Q)$, are shown in Figures 4 and 5.

In Fig. 5, we show our results for $I_{e l}(Q)$ and $I_{M F F}(Q)$ at $34 \mathrm{GPa}$ and $2000 \mathrm{~K}$, as well as the total x-ray scattering intensity calculated using standard form factors, $I_{s t d}(Q)$. We observed nearly exact agreement between $I_{e l}(Q)$ and $I_{M F F}(Q)$ for all densities investigated at 1500 and $2000 \mathrm{~K}$. The $I_{s t d}(Q)$ provided reasonable agreement with $I_{e l}(Q)$ at the lower densities investigated, but the agreement got increasingly worse as the density increased. The results for our fits of the parameters $\alpha$ and $\delta$ are shown in Fig. 4. It is notable that the results for both parameters show little dependence on temperature and an approximately linear dependence pressure. This can allow for easy extrapolation of values for interpretation of high temperaturepressure $\mathrm{x}$-ray experiments.

The fact that $\alpha$ increases monotonically with pressure and $\delta$ decreases even more sharply implies that as the superionic solid is formed, the charges on the oxygens are increasing. In Fig. 6, we have calculated the Mulliken charge distribution [32] for oxygen and hydrogen, and we see that the charges similarly increase and decrease monotonically, respectively. The fact that the fitted values of $\delta$ decrease monotonically 
simultaneously implies that the electron distribution about the oxygens is becoming less diffuse as a function of pressure. This implies that as the superionic solid is formed, water begins to resemble an ensemble of closely packed charges. However, confirmation of such an idea would require detailed electronic structure calculations, and is beyond the scope of this work.

Finally, we have computed the oxygen radial distribution function in order to examine the effects of using our modified form factors vs. using standard ones. The results for $34 \mathrm{GPa}$ and $2000 \mathrm{~K}$ are shown in Fig. 7. The $g\left(R_{O O}\right)$ was determined by taking the total x-ray scattering intensity computed from QUICKSTEP, $I_{e l}(Q)$, and making the approximation that the entire intensity is due to the oxygens alone. Consequently, we were able to determine the oxygen structure factor $S_{O O}(Q)$ for each type of form factor. The $g\left(R_{O O}\right)$ was then determined by taking the Fourier Transform, as mentioned above. As shown in the figure, at the above state point, the use of different form factors yields only slight changes in the RDF. The same is even true at much higher pressures, viz., $115 \mathrm{GPa}$, where the value of $\alpha$ is relatively high (ca. 1.21). Thus, in general it appears that using the standard form factors could yield reasonably accurate RDFs.

\section{Conclusion}

We have a great new capability with electron structure codes such as CP2K to calculate the total $\mathrm{x}$-ray scattering intensity of water under extreme thermodynamic conditions. The $I(Q)$ is a very sensitive probe at elevated pressure and temperature conditions, where changes in the electronic structure of water can vary rapidly with changing thermodynamic conditions. As a result, it can be used to facilitate better comparison between experiment and theory, where exact matching of thermodynamic conditions can be quite difficult. This could yield improvements in equation of state modeling of water at high pressures and temperatures.

We observe that standard form factors can be used for reasonably accurate determination of the $g\left(R_{O O}\right)$. However, a more accurate $S(Q)$ could be essential for analysis of specific spectral peaks, as discussed below. Such studies would greatly benefit from more accurate atomic form factors. Our data in Fig. 4 could easily be fit to a linear model and extrapolated to the pressure and temperature of interest. The values of our fitted parameters and the apparent accuracy of standard atomic form factors indicates that covalent bonding at extreme thermodynamic conditions is largely weakened. Thus, one can think of water at high pressure and temperature as an ensemble of closely packed ionic charges.

The $I(Q)$ serves as an excellent order parameter in that it can distinguish ready between phases of water, such as the molecular liquid and superionic solid. We have seen that in one simulation the $I(Q)$ shows the presence of an oxygen bcc lattice where other standard statistical mechanical tools (viz., diffusion constant, radial distribution function, etc.) did not [2]. Through analysis of the 111 reflection, we present a possi- 
ble method for detecting the presence of superionic hydrogen diffusion. One can use simulations and our modified atomic form factors to fit to experimental data in order to extract accurate values for the $S(Q)$. Subsequently, the fraction of the $I_{111}$ due to hydrogen-hydrogen scattering as a function of pressure and temperature could be determined. Since the decrease in the hydrogen contribution is likely due to the sharply its sharply increased mobility, the superionic phase boundary could consequently be observed experimentally.

This work was performed under the auspices of the U. S. Department of Energy by University of California, Lawrence Livermore National Laboratory under contract W-7405-Eng-48. 


\section{References}

[1] A. F. Goncharov, N. Goldman, L. E. Fried, J. C. Crowhurst, I.-F. W. Kuo, C. J. Mundy, and J. M. Zaug, Phys. Rev. Lett. 94, 125508 (2005).

[2] N. Goldman, L. E. Fried, I.-F. W. Kuo, and C. J. Mundy, Phys. Rev. Lett. 94, 217801 (2005).

[3] N. Goldman and L. E. Fried, J. Chem. Phys. 125, 044501 (2006).

[4] J. M. Sorenson, G. Hura, R. M. Glaeser, and T. Head-Gordon, J. Chem. Phys. 113, 9149 (2000).

[5] G. Hura, J. M. Sorenson, R. M. Glaeser, and T. Head-Gordon, J. Chem. Phys. 113, 9140 (2000).

[6] M. Krack, A. Gambirasio, and M. Parrinello, J. Chem. Phys. 117, 9409 (2002).

[7] G. Hura, D. Russo, R. M. Glaeser, T. Head-Gordon, M. Krack, and M. Parrinello, Phys. Chem. Chem. Phys. 5, 1981 (2003).

[8] R. J. Hemley, A. P. Jephcoat, H. K. Mao, C. S. Zha, L. W. Finger, and D. E. Cox, Nature 330, 737 (1987).

[9] Y. Fei, H. Mao, and R. J. Hemley, J. Chem. Phys. 99, 5369 (1993).

[10] E. Wolanin, P. Pruzan, J. C. Chervin, B. Canny, M. Gauthier, D. Häusermann, and M. Hanfland, Phys. Rev. B 56, 5781 (1997).

[11] Y. Yoshimura, S. T. Stewart, M. Somayazulu, and R. J. Hemley, J. Chem. Phys. 124, 024502 (2006).

[12] P. Loubeyre, R. LeToullec, E. Wolanin, M. Hanfland, and D. Hausermann, Nature 397, 503 (1999).

[13] C. Bina and A. Navrotsky, Nature 408, 844 (2000).

[14] N. S. Gingrich, Rev. Mod. Phys. 15, 90 (1943).

[15] International Table for X-ray Crystallography, 3rd ed., edited by J. A. Ibers and W. C. Hamilton (Kluwer Academic, Dordrecht, 1989), Vol. 3.

[16] R. J. Hemley, Ann. Rev. Phys. Chem. 51, 763 (2000).

[17] J. VandeVondele, M. Krack, F. Mohamed, M. Parrinello, T. Chassaing, and J. Hutter, Comp. Phys. Comm. 167, 103 (2005).

[18] M. P. Allen and D. J. Tildesley, Computer Simulation of Liquids (Clarendon Press, Oxford, 1987). 
[19] A. D. Becke, Phys. Rev. A 38, 3098 (1988).

[20] C. Lee, W. Yang, and R. G. Parr, Phys. Rev. B 37, 785 (1988).

[21] N. Troullier and J. Martins, Phys. Rev. B 43, 1993 (1991).

[22] W. G. Hoover, Phys. Rev. A 31, 1695 (1985).

[23] J. Grossman, E. Schwegler, E. W. Draeger, F. Gygi, and G. Galli, J. Chem. Phys. 120, 300 (2004).

[24] I.-F. Kuo, C. Mundy, M. McGrath, J. Siepmann, J. VandeVondele, M. Sprik, J. Hutter, B. Chen, M. Klein, F. Mohamed, M. Krack, and M. Parrinello, J. Phys. Chem. B 108, 12990 (2004).

[25] J. Krog-Moe, Acta Crystallogr. 9, 951 (1956).

[26] N. Norman, Acta Crystallogr. 10, 370 (1957).

[27] J. Wang, A. N. Tripathi, and J. V. H. Smith, J. Chem. Phys. 101, 4842 (1994).

[28] N. Watanabe, S. Ten-no, S. Pal, S. Iwata, and Y. Udagawa, J. Chem. Phys. 111, 827 (1999).

[29] L. R. M. Morin, J. Phys. Chem. Ref. Data 11, 1091 (1982).

[30] For information of the CP2K code see http://www.berlios.de.

[31] W. H. Press, S. A. Teukolsky, W. T. Vetterling, and B. P. Flannery, Numerical Recipes in Fortran 77 (Cambridge University Press, Cambridge, 1992).

[32] R. S. Mulliken, J. Chem. Phys. 23, 1833 (1955). 


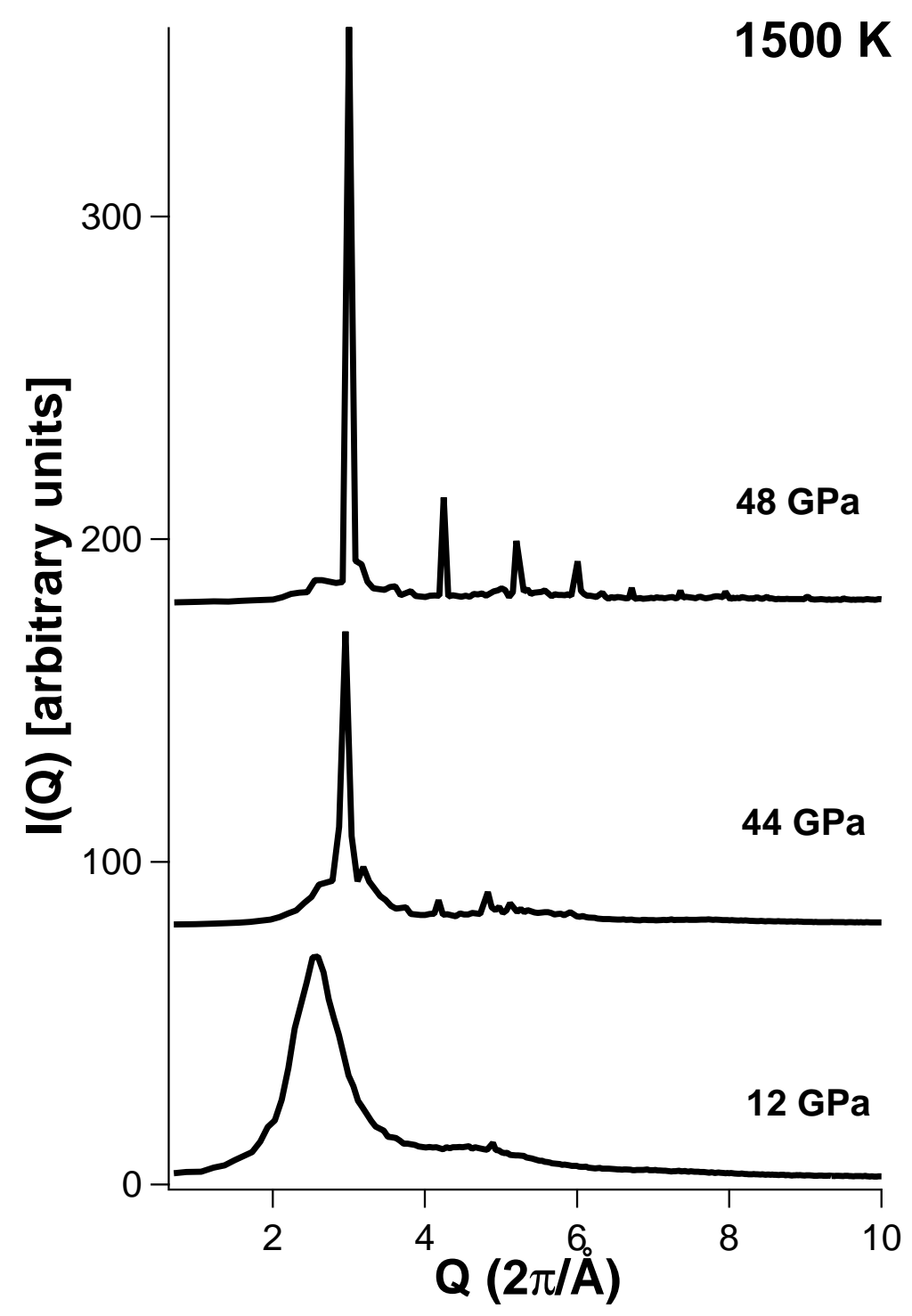

Figure 1: $I(Q)$ for water compressed at $1500 \mathrm{~K}$. 


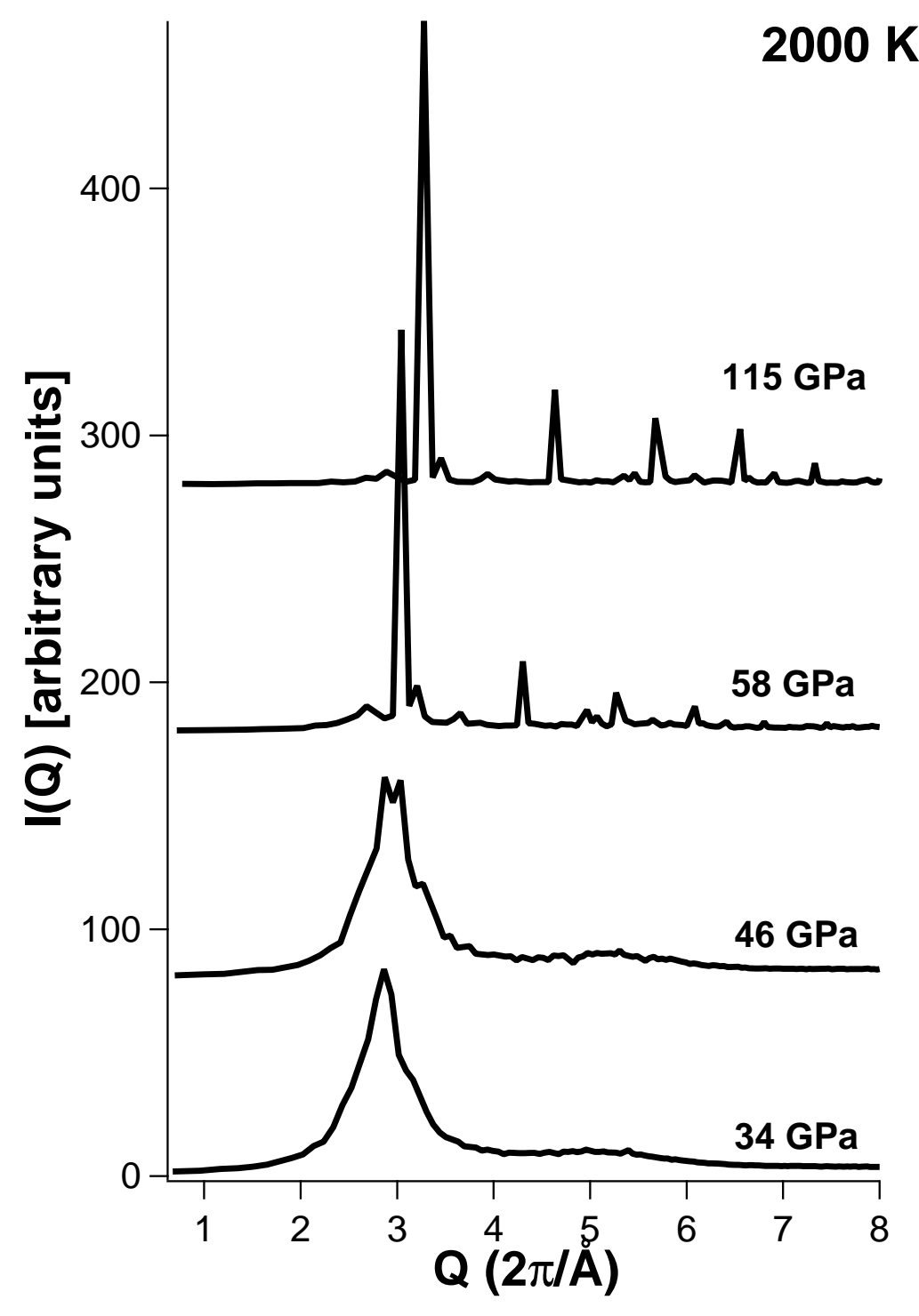

Figure 2: $I(Q)$ ice VII simulations heated to $2000 \mathrm{~K}$. 


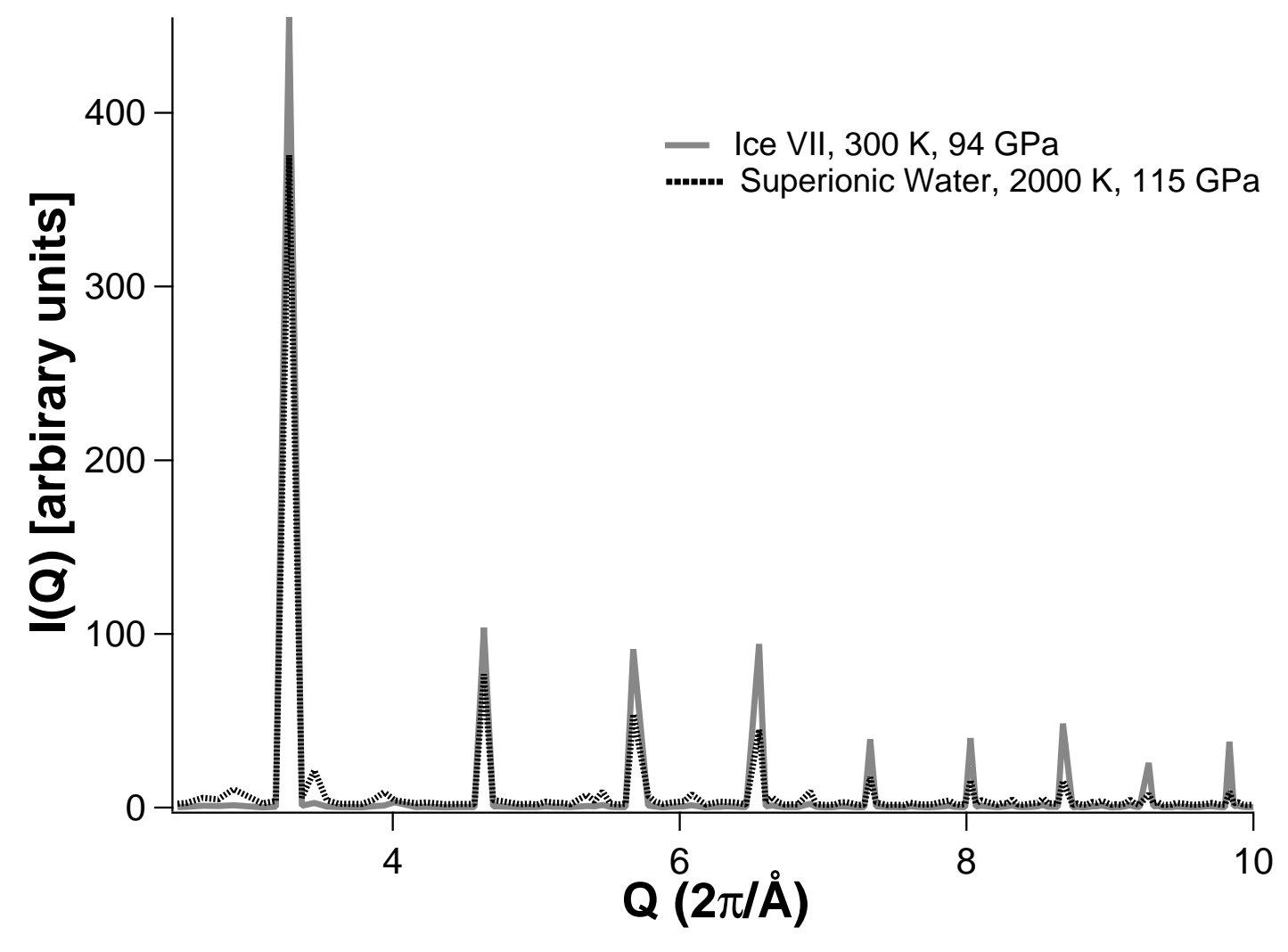

Figure 3: Comparison of the total x-ray scattering intensity of ice VII to the symmetrically hydrogen bonded form of superionic water. It was difficult to make a direct comparison of our spectrum of ice VII (95 GPa and $300 \mathrm{~K}$ ) to experiment due to the fact that only a few studies exist at the same conditions $[8,10,12]$, and none publish detailed spectral features at ca. $\mathrm{P}=100 \mathrm{GPa}$. Nonetheless, we find that our results yields an equation of state and lattice cell parameter within ca. $10 \%$ of experimental results $[8,10]$. 


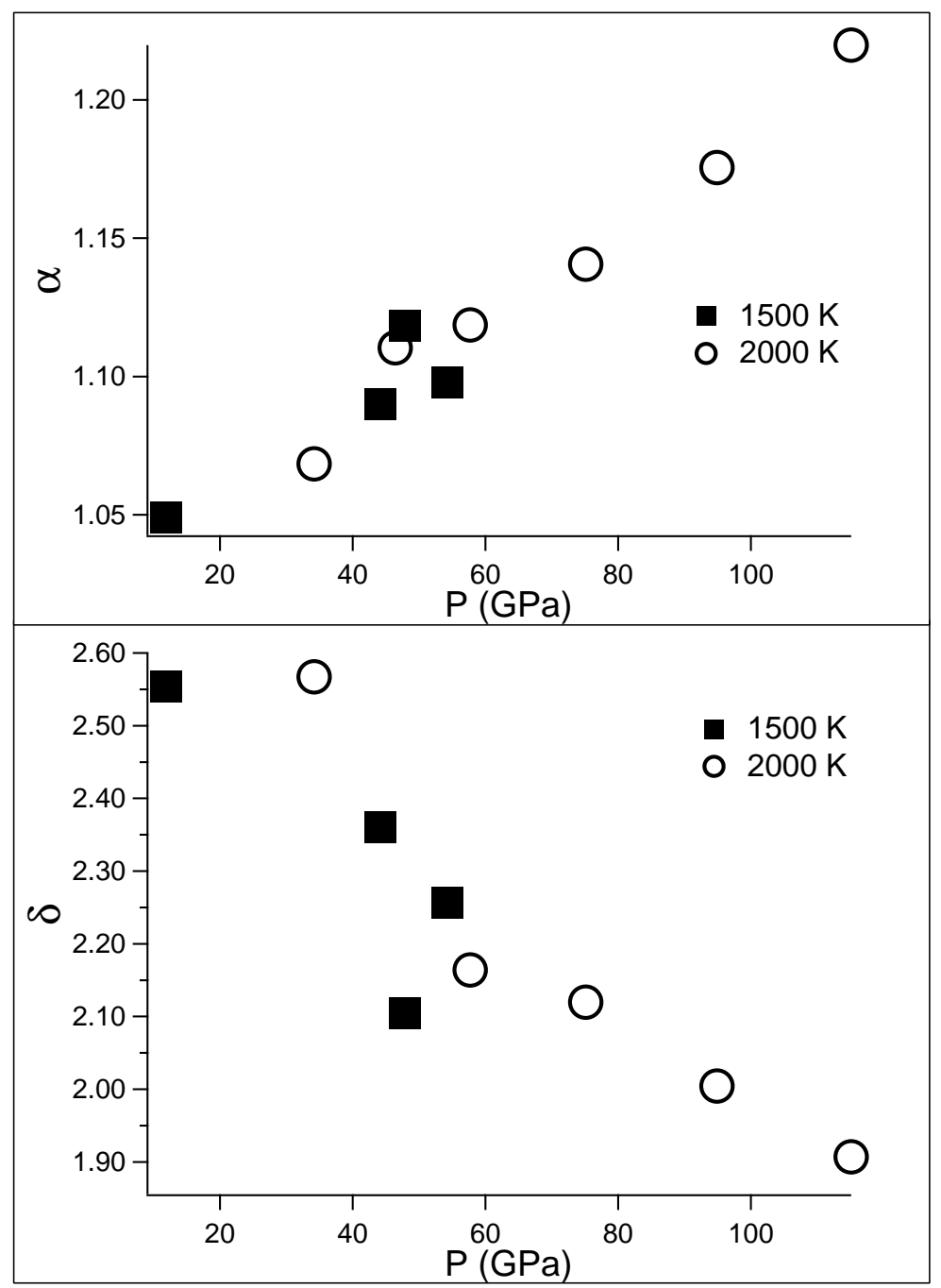

Figure 4: Results for 1500 and $2000 \mathrm{~K}$ of our best fit of $\alpha$ and $\delta$ for the modified form factors from Equation 6. 


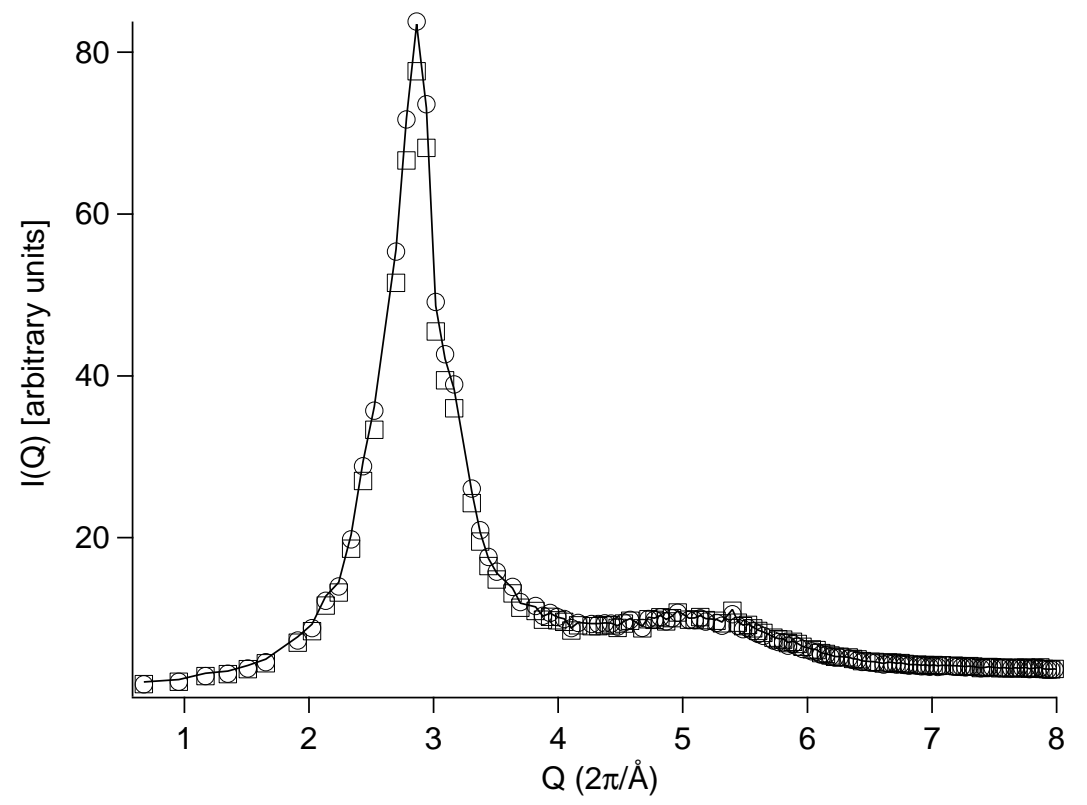

(a)

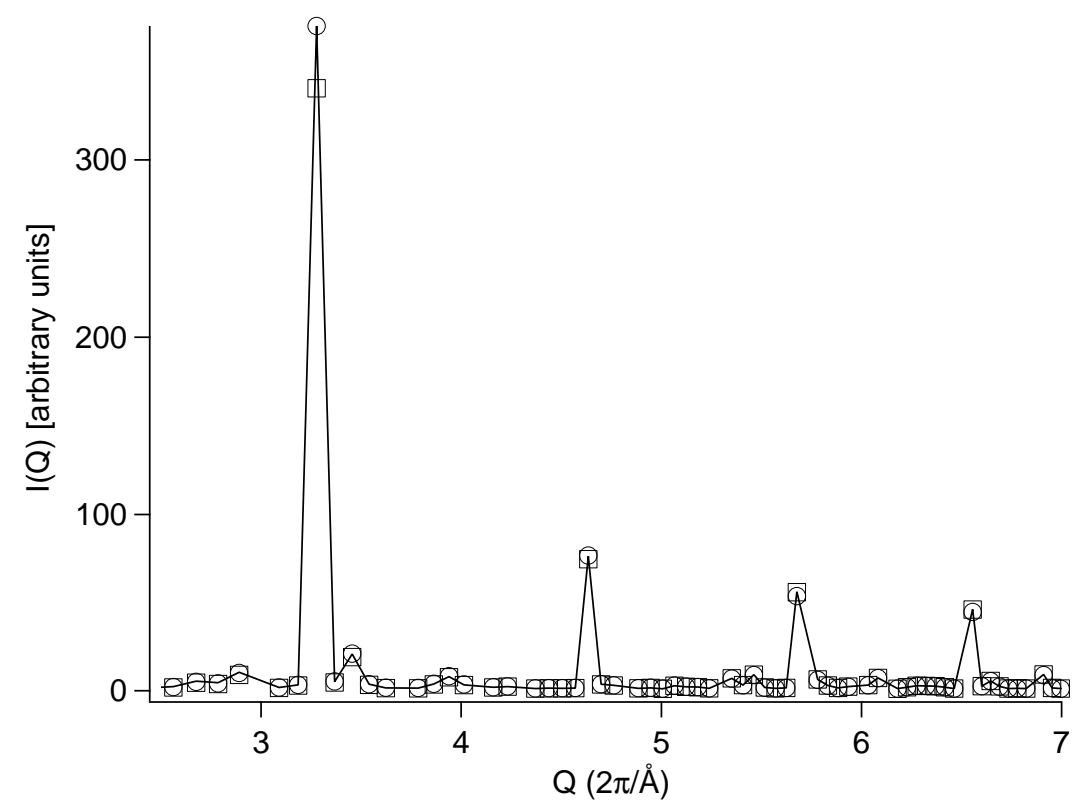

(b)

Figure 5: Comparison of computed $I_{e l}(Q)$ at $34 \mathrm{GPa}$ and $2000 \mathrm{~K}$. The dashed line corresponds to the result from the standard atomic form factors, and the solid line to the modified form factors with fitted values of $\alpha$ and $\delta$. The '॰' correspond to our result from QUICKSTEP. 


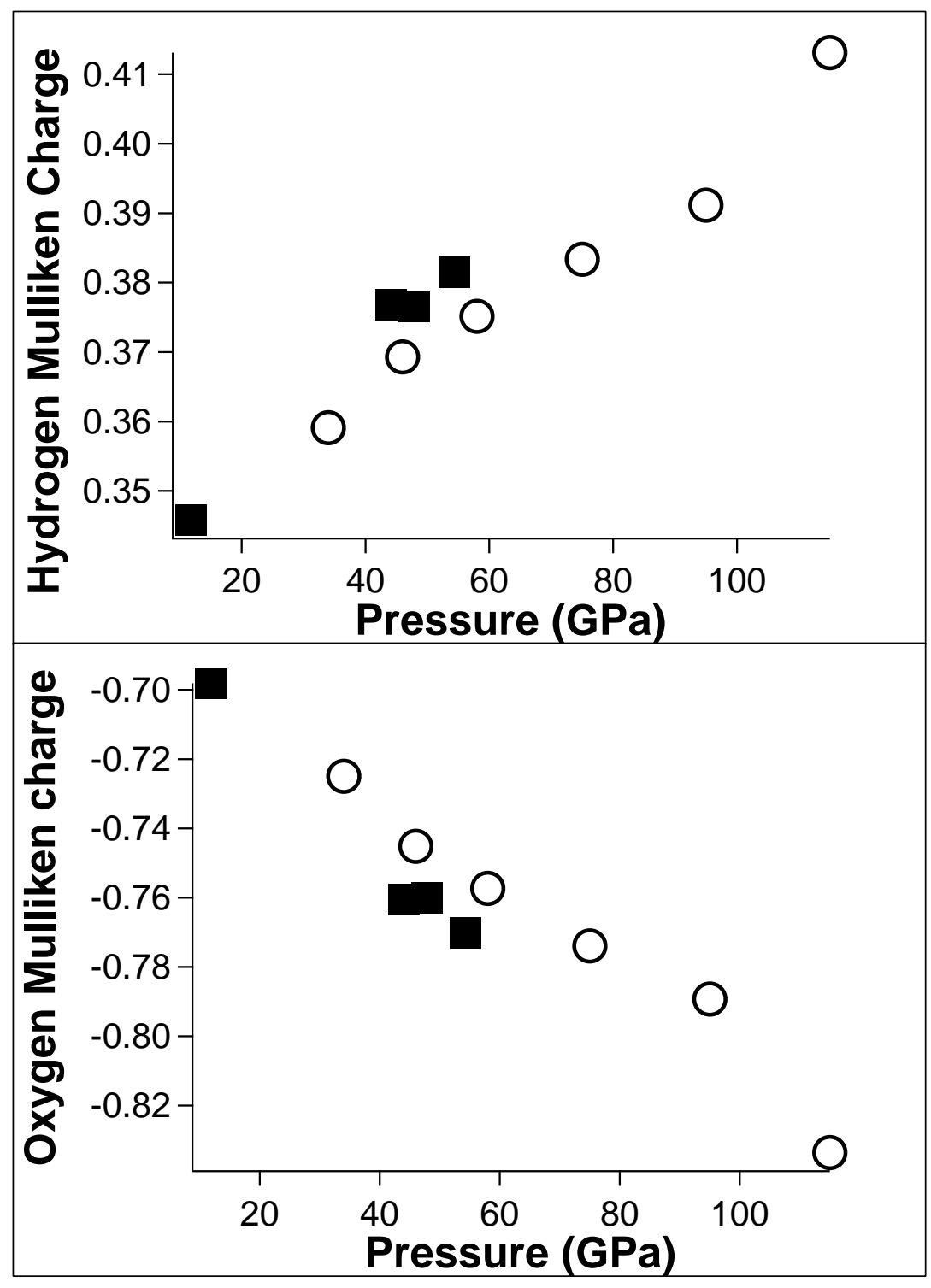

Figure 6: Mulliken charges for hydrogen and oxygen for all simulations. 


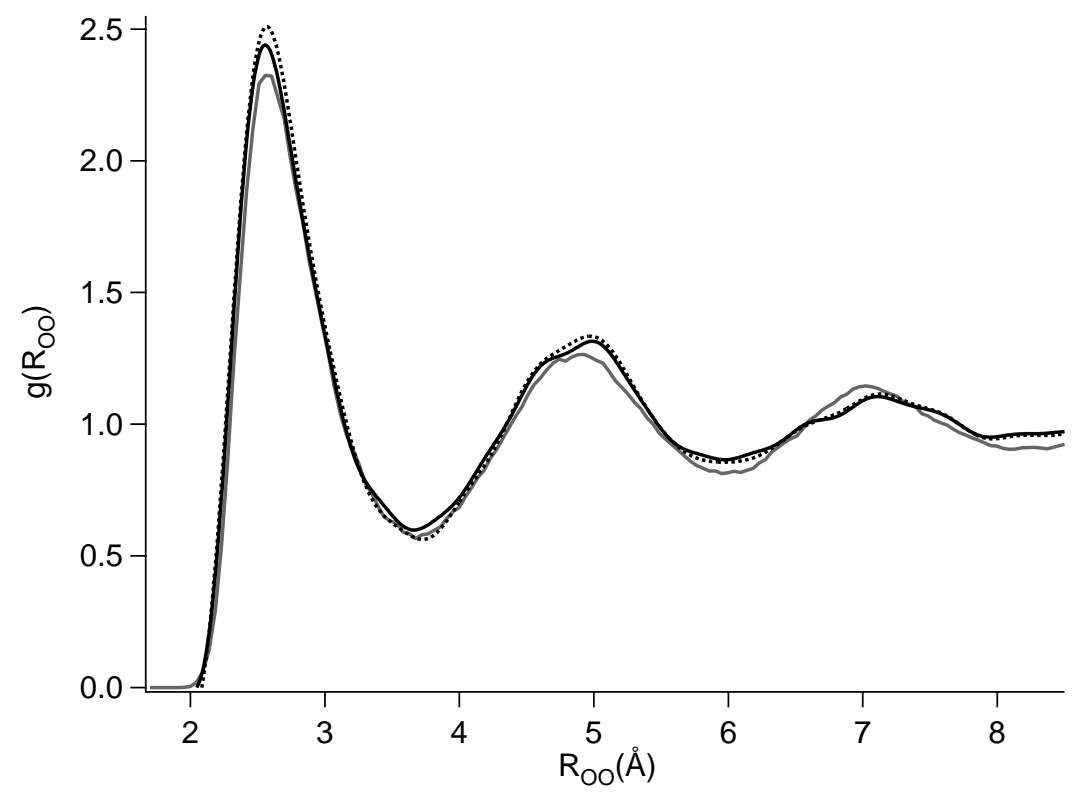

Figure 7: Oxygen-oxygen radial distribution functions at $34 \mathrm{GPa}$ and $2000 \mathrm{~K}$. The dashed line corresponds to the Fourier Transform of the $\mathrm{S}(\mathrm{Q})$ from the standard atomic form factor, and the solid black line to the Fourier Transform from the $\mathrm{S}(\mathrm{Q})$ form our modified form factor. The gray line corresponds to the result from the CPMD simulation. 